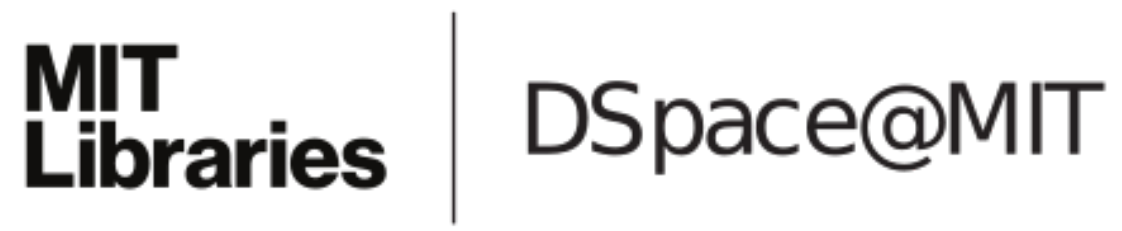

\author{
MIT Open Access Articles
}

\begin{abstract}
Non-rigid registration of $3 D$ ultrasound for neurosurgery using automatic feature detection and matching
\end{abstract}

The MIT Faculty has made this article openly available. Please share how this access benefits you. Your story matters.

Citation: Machado, Inês et al. "Non-rigid registration of 3D ultrasound for neurosurgery using automatic feature detection and matching." International Journal of Computer Assisted Radiology and Surgery, vol. 13, no. 10, 2018, pp. 1525-1538 (c) 2018 The Author(s)

As Published: $10.1007 / \mathrm{S11548-018-1786-7}$

Publisher: Springer Nature

Persistent URL: https://hdl.handle.net/1721.1/126069

Version: Author's final manuscript: final author's manuscript post peer review, without publisher's formatting or copy editing

Terms of use: Creative Commons Attribution-Noncommercial-Share Alike 


\title{
Non-rigid registration of 3D ultrasound for neurosurgery using automatic feature detection and matching
}

\author{
Inês Machado ${ }^{1,2}$, Matthew Toews ${ }^{3}$, Jie Luo ${ }^{1,4}$, Prashin Unadkat ${ }^{5}$, Walid Essayed ${ }^{5}$, Elizabeth \\ George $^{1}$, Pedro Teodoro ${ }^{2}$, Herculano Carvalho ${ }^{6}$, Jorge Martins ${ }^{2}$, Polina Golland ${ }^{7}$, Steve \\ Pieper $^{1,8}$, Sarah Frisken ${ }^{1}$, Alexandra Golby ${ }^{5}$, and William Wells III ${ }^{1,7}$ \\ ${ }^{1}$ Department of Radiology, Brigham and Women's Hospital, Harvard Medical School, 75 Francis \\ St., Boston, MA 02115, USA \\ 2IDMEC, Instituto Superior Técnico, Universidade de Lisboa, Av. Rovisco Pais 1, 1049-001 \\ Lisbon, Portugal \\ 3École de Technologie Superieure, 1100 Notre-Dame St W, Montreal QC H3C 1K3, Canada \\ ${ }^{4}$ Graduate School of Frontier Sciences, University of Tokyo, 5-1-5 Kashiwanoha, Kashiwa, Chiba, \\ Japan \\ ${ }^{5}$ Department of Neurosurgery, Brigham and Women's Hospital, Harvard Medical School, 75 \\ Francis St., Boston, MA 02115, USA \\ ${ }^{6}$ Department of Neurosurgery, CHLN, Hospital de Santa Maria, Avenida Professor Egas Moniz, \\ 1649-035 Lisbon, Portugal \\ ${ }^{7}$ Computer Science and Artificial Intelligence Laboratory, Massachusetts Institute of Technology, \\ 32 Vassar St, Cambridge, MA 02139, USA \\ 8Isomics, Inc., 55 Kirkland St, Cambridge, MA 02138, USA
}

\begin{abstract}
Purpose-The brain undergoes significant structural change over the course of neurosurgery, including highly nonlinear deformation and resection. It can be informative to recover the spatial mapping between structures identified in preoperative surgical planning and the intraoperative state of the brain. We present a novel feature-based method for achieving robust, fully automatic deformable registration of intraoperative neurosurgical ultrasound images.
\end{abstract}

Methods-A sparse set of local image feature correspondences is first estimated between ultrasound image pairs, after which rigid, affine and thin-plate spline models are used to estimate dense mappings throughout the image. Correspondences are derived from 3D features, distinctive generic image patterns that are automatically extracted from 3D ultrasound images and

Correspondence to: Inês Machado.

Compliance with ethical standards

Conflict of interest The authors declare that they have no conflict of interest.

Ethical approval All procedures performed in studies involving human participants were in accordance with the ethical standards of the institutional and/or national research committee and with the 1964 Helsinki Declaration and its later amendments or comparable ethical standards.

Informed consent Informed consent was obtained from all individual participants included in the study. 
characterized in terms of their geometry (i.e., location, scale, and orientation) and a descriptor of local image appearance. Feature correspondences between ultrasound images are achieved based on a nearest-neighbor descriptor matching and probabilistic voting model similar to the Hough transform.

Results-Experiments demonstrate our method on intraoperative ultrasound images acquired before and after opening of the dura mater, during resection and after resection in nine clinical cases. A total of 1620 automatically extracted 3D feature correspondences were manually validated by eleven experts and used to guide the registration. Then, using manually labeled corresponding landmarks in the pre- and post-resection ultrasound images, we show that our feature-based registration reduces the mean target registration error from an initial value of 3.3 to $1.5 \mathrm{~mm}$.

Conclusions-This result demonstrates that the 3D features promise to offer a robust and accurate solution for 3D ultrasound registration and to correct for brain shift in image-guided neurosurgery.

\section{Keywords}

Brain shift; Intraoperative ultrasound; Image-guided neurosurgery; Image registration; 3D scaleinvariant features

\section{Introduction}

Neuronavigation systems can be used to determine the position of brain tumors during surgical procedures relative to preoperative imaging, typically magnetic resonance (MR) images [1]. Commercial systems employ an electromagnetic or optical device to track the surgical tools and model the patient's head and its content as a rigid body. Paired-point registration based on anatomical features or surface-based registration methods are commonly used to determine the rigid body transformation from the coordinate system of the preoperative image to the coordinate system of the patient during surgery. During surgery, cerebrospinal fluid drainage, use of diuretics and tumor resection cause the brain to deform and therefore invalidate the estimated rigid transformation [2]. Brain deformation during surgery, known as brain shift, along with registration and tracking errors reduces the accuracy of image-guided neurosurgery based on neuronavigation systems [3-5].

Because maximum safe resection is the single greatest modifiable determinant of survival [6] and strongly correlated with prognosis in patients with both low-grade [7] and highgrade gliomas [8], the development of intraoperative imaging techniques is desirable because they guide the surgeon toward obtaining a more complete resection while helping to prevent damage to normal brain [9].

Intraoperative magnetic resonance (iMR) imaging has been used during surgery offering high contrast for visualization of tissues using multiple sequences [10-13]. However, MR devices are expensive and require a dedicated operating room and specialized non-magnetic tools making this technology unavailable in most centers worldwide. iMR is also timeconsuming, adding an hour or more per scan to a surgical intervention. 
On the other hand, intraoperative ultrasound (iUS) appears to be a promising technology to compensate for brain shift [14-17]. iUS is relatively inexpensive and does not require changes to the operating room. However, awareness of artifacts in ultrasound images that may occur during tumor resection is a necessity for successful and safe surgery when using iUS for resection control.

Registration of intraoperative imaging is challenging, especially of iUS images. Brain ultrasound images acquired prior to resection often have high image quality, providing the capability of localizing surgical targets, typically defined in the preoperative MR during surgical planning. During surgery, e.g., right after opening the dura membrane, this capability degrades as artifacts may occur. Moreover, brain deformation is a complex and spatiotemporal phenomenon [3], requiring non-rigid registration algorithms capable of mapping structures in one image that have no correspondences in a second image, such as the tumor and resection site [18].

A solution is to use biomechanical modeling to combine intraoperatively acquired data with computational models of brain deformation to update preoperative images during surgery [19-22]. Development of accurate biomechanical models is important to compensate for brain displacement and is an ongoing focus of a large portion of the research community. However, most of the research work that has been done in biomechanical modeling of the brain tissue for this purpose is not completely independent of the registration methods.

We investigate the use of intraoperative 3D ultrasound to compensate for brain shift during neurosurgical procedures.

A body of prior work has investigated iUS image registration in the context of neurosurgery [23-25]. For reducing computational complexity, deformable registration approaches typically adopt iterative algorithms that are only guaranteed to converge to locally optimal solution. They are thus prone to identifying erroneous, suboptimal solutions if not initialized correctly, particularly if there is no smooth geometric transformation that models the differences between the images, e.g., following brain tumor resection. These methods may require initialization within a "capture range" of the correct solution, where initialization is typically provided via external labels or segmentation of regions of interest within ultrasound images and the alignment is optimized primarily for the specific labels [26,27]. Alternatively, generic salient image features have been applied using a wide variety of methods [28-32], and distinctive local neighborhoods surrounding edges and texture features can be used to identify image-to-image correspondences prior to registration [33]. While correspondences between distinctive local features may be achieved, a challenge remains when computing registration throughout the image space, particularly in regions where one-to-one correspondence is ambiguous or nonexistent.

A major advance in image recognition was the development of invariant feature detectors, capable of reliably identifying the same distinctive image features in a manner independent of geometrical deformations. In particular, scale-invariant features (SIFT) are invariant to translation, rotation and scale changes and provide robust feature matching across a substantial range of addition of noise and changes in illumination [34]. SIFT features were 
extended to 3D imaging with the 3D SIFT-Rank algorithm [35]. 3D SIFT-Rank matching was previously used to stitch multiple hepatic ultrasound volumes into a single panoramic image of a health liver [36]. Ultrasound volumes were acquired from healthy subjects with a breath holding protocol; thus, soft tissue deformation is minimal and modeled as rigid. Additionally, keypoints are encoded using a 2048-element 3D SIFT descriptor, whereas the memory efficient 64-element SIFT-Rank descriptor was adopted. 3D SIFT-Rank was also used to align left and right ventricle volumes in 4D cardiac ultrasound sequences to enlarge the field of view [37]. Correspondences are identified between 3D volumes at similar points in the cardiac cycle, where deformation is minimal and approximately rigid.

In this paper, feature-based registration aims to identify a globally optimal spatial mapping between 3D iUS images, based on a sparse set of scale-invariant feature correspondences. We demonstrate the application of a fully automated feature extraction and matching [35] for iUS registration to compensate for brain shift. To the best of our knowledge, it is the first work to apply 3D SIFT-Rank features to iUS registration in image-guided neurosurgery.

\section{Methods}

3D ultrasound images consist of image patterns that are challenging to localize or register across datasets. For efficient and robust registration, we adopt a local feature-based registration strategy, where the image is represented as a collection of scale-invariant features, generic salient image patches that can be identified and matched reliably between different images of the same underlying object. Feature-based registration operates by identifying a set of pairwise feature correspondences between a pair of images to be registered based on local appearance descriptor similarity, after which feature geometry is used to prune spurious, incorrect correspondences via the generalized Hough transform. Feature correspondences were manually validated by radiologist and non-radiologist physicians. Figure 1 presents a summary of the proposed feature-based registration for intraoperative 3D ultrasound.

\section{Feature extraction}

We use the 3D SIFT-Rank algorithm [35] to automatically extract and match features from 3D iUS. Feature extraction seeks to identify a set of salient image features from a single image. Each feature $f=\{g, \bar{a}\}$ is characterized by geometry $g=\{\bar{x}, \sigma, \theta\}$ and appearance descriptor $\bar{a}$. Feature geometry consists of a 3D location $\bar{x}=(x, y, z)$, a scale $\sigma$, and a local orientation specified by a set of three orthonormal unit vectors $\theta=\left\{\hat{\theta}_{1}, \hat{\theta}_{2}, \hat{\theta}_{3}\right\}$. Appearance descriptor $\bar{a}$ is a vector that encodes the local image appearance. Feature extraction operates first by (1) detecting the location and scale of distinctive image regions and then (2) encoding the appearance of the image within the region. In general, feature detection involves identifying a set of distinctive regions $\left\{\left(\bar{x}_{i}, \sigma_{i}\right)\right\}$ that maximize a function of local image saliency, e.g., entropy [38] and Gaussian derivative magnitude [39]. Following the SIFT method, we identify regions as extrema of the difference-of-Gaussian (DoG) function, given by Eq. (1). 


$$
\left\{\left(\bar{x}_{i}, \sigma_{i}\right)\right\}=\text { local } \underset{\vec{x}, \sigma}{\operatorname{argmax}}|f(\bar{x}, k \sigma)-f(\bar{x}, \sigma)|
$$

where $f(\bar{X}, \sigma)=I(\bar{X}) * G(\bar{X}, \sigma)$ represents the convolution of image $I(\bar{X})$ with a Gaussian kernel $G(\bar{x}, \sigma)$ of variance $\sigma^{2}$ and $k$ is a multiplicative sampling rate in scale $\sigma$.

Once a set of features has been detected, the image content surrounding each region $\left(\bar{x}_{i}, \sigma_{i}\right)$ is encoded as a local appearance descriptor $\bar{a}_{i}$ to compute feature correspondences between images. We adopt the 3D SIFT-Rank descriptor [35], where the image content about each region $\left(\bar{x}_{i}, \sigma_{i}\right)$ is first cropped and normalized to a $11 \times 11 \times 11$ voxel patch, after which local image gradients are grouped into a 64-element descriptor $\bar{a}_{i}$ based $8=2 \times 2 \times 2$ spatial bins and 8 orientation bins. Each image gradient sample increments a bin defined by location and orientation, and the bin is incremented by the gradient magnitude. Finally, this vector is rank ordered, where each element of $\bar{a}_{i}$ takes on its index in a sorted array rather than the original gradient histogram bin value. Rank ordering provides invariance to monotonic deformations of image gradients and results in more accurate nearest-neighbormatched feature [40].

\section{Feature matching}

Let $T: F \rightarrow M$ be a spatial mapping between feature sets $F=\left\{f_{i}\right\}$ and $M=\left\{f_{j}\right\}$ in fixed and moving images, respectively. Feature-based registration seeks a maximum a posteriori (MAP) transform $T^{*}=\operatorname{argmax}_{T}\{p(T \mid F, M)\}$, which can be achieved by a 2-step image-toimage feature matching process. First, a set of candidate feature correspondences $\left(f_{i}, f_{j}\right)$ are identified between all features in the moving image and the fixed image based on minimum appearance descriptor difference. Descriptor difference is evaluated via the Euclidean distance $d\left(\bar{a}_{i}, \bar{a}_{j}\right)=\left\|\bar{a}_{i}-\bar{a}_{j}\right\|$. Second, for each candidate matching feature pair $\left(f_{i}, f_{j}\right)$, a hypothesis $T_{i j}: \mathrm{g}_{i} \rightarrow \mathrm{g}_{j}$ is estimated as to an approximate linear transform between images based on geometry descriptors $\left(\mathrm{g}_{i}, \mathrm{~g}_{j}\right)$. This can be viewed as maximizing the likelihood $p\left(g_{i}\right.$ $\left.\mid T_{i j}, g_{j}\right)$, and as each feature incorporates a position, orientation and scale, a single pair of features contains sufficient information for estimating a scaled rigid transform hypothesis (or linear similarity transform) between images. Transform hypotheses estimated from all features pairs are generated and then grouped to identify a dense cluster of geometrically consistent pairs/transforms that are "inliers" of a single global transform at a specified tolerance level, where clustering is achieved via the robust mean-shift algorithm [28]. Intuitively, the transform hypothesis supported by the highest number of inliers represents the MAP transform $T^{*}$, given a Gaussian mixture model over the appearance and geometry of individual features. The result of the matching process is the MAP transform $T^{*}$, along with a set of inlier feature pairs represent valid image-to-image correspondences.

\section{Feature-based registration}

Deformable registration algorithms must often be initialized near the correct solution where initialization may take the form of an approximate, initial rigid or affine registration [41]. A 12-parameter affine transformation was used to find a spatial transform that maps two ultrasound images. The most widely applied method for landmark-based non-rigid 
registration is based on thin-plate splines as they have regularization properties that are, at least loosely, inspired by mechanics $[42,43]$. Thin-plate splines were used to get the deformation field between two ultrasound images.

\section{Experiments}

\section{Patients}

Nine patients ( 3 females, 6 males; mean age 44 years) scheduled for resection of suspected/ known primary or metastatic brain tumor in a multi-modality image-guided surgical suite [44] were included in this study. After histologic examination, it was determined that 4 patients had low-grade gliomas, 4 had high-grade gliomas, and 1 had metastatic brain tumor. Mean tumor volume was $19.5 \mathrm{~cm}^{3}$, ranging from 0.1 to $57.0 \mathrm{~cm}^{3}$. Two of the cases were first operations, and seven were reoperations. All patients provided informed consent. Clinical details, including the demographics, pathologic diagnosis, volume and location of tumor, are shown in Table 1.

\section{MR data acquisition}

For preoperative planning and integration into the neuronavigation system, images were acquired on a 3-Tesla MR scanner after the administration of $0.1-0.2 \mathrm{mmol} / \mathrm{kg}$ of Gadabutrol (Gadavist, Bayer Schering Pharma, Germany). Intraoperative MR was performed on a 3-Tesla MR scanner (Siemens Healthcare GmbH, Erlangen, Germany). Postoperative brain MR protocols were identical to the preoperative acquisitions. Two patients did not have postoperative MR. Tumor volume was calculated on the iPlan Cranial 3.0 software (Brainlab, Munich, Germany) using the contrast enhancing portion of the tumor.

Figure 2 shows the representative axial slices ( $\mathrm{T} 2$ or $\mathrm{T} 1$ post-contrast) from the preoperative MR of the nine subjects.

\section{US data acquisition}

During surgery, two freehand ultrasound sweeps were acquired, before and after the opening of the dura membrane but before inducing other structural changes. After the first two acquisitions, the surgeon resected the tumor until what he thought was the maximum possible resection. For some patients, it was also possible to acquire iUS images during resection and prior to intraoperative MR. The ultrasound images were acquired by two neurosurgeons (attending and clinical fellow) with significant expertise in the use of intraoperative tracked 3D ultrasound as it is a routine part of neurosurgical procedures in our institution.

The position of the ultrasound probe relative to the patient's head was monitored via optical tracking using the VectorVision Sky neuronavigation system (target registration error equal to $1.13 \pm 0.05 \mathrm{~mm}$ ) (Brainlab, Munich, Germany) [45]. A touch-based pointer (Softouch, BrainLab) was used to collect a cloud of points from the surface of the head and facial region. A surface-based registration was used to determine the rigid body transformation from the coordinate system of the preoperative image to the coordinate system of the patient. 
Intraoperative ultrasound was acquired on a BK Ultrasound 3000 system (BK Medical, Analogic Corporation, Peabody, USA) that is directly connected to the Brainlab neuronavigation system. The BK craniotomy probe 8861 was used in the scanning B-mode with frequency range of $10-3.8 \mathrm{MHz}$. The stylus calibration error of the probe is less than $0.5 \mathrm{~mm}$.

An Epiphan USB video grabber was used to capture iUS images from the BK monitor. Image data were imported into 3D Slicer using OpenIGTLink [46] and reconstructed as 3D volumes using the PLUS library [47]. The volume reconstruction method in PLUS is based on the work of $[48,49]$. The first step of the procedure is the insertion of 2D image slices into a 3D volume. This is implemented by iterating through each pixel of the rectangular or fan-shaped region of the slice and distributing the pixel value in the closest 8 volume voxels. The voxel value is determined as a weighted average of all coinciding pixels. Slice insertion is performed at the rate the images are acquired; therefore, individual image slices do not have to be stored and the reconstructed volume is readily available at the end of the acquisition. Typically, sweeps contained between 100 and 300 frames of 2D ultrasound data and were reconstructed at a voxel size of $0.5 \times 0.5 \times 0.5 \mathrm{~mm}^{3}$. A typical example of pre- and partial post-resection ultrasound images and their initial misalignments is shown in Fig. 3. As most brain tumors have higher mass density and sound velocity than the surrounding normal brain [50], the ultrasound images can result in sharp interfaces as shown in Fig. 3.

\section{Feature extraction and matching}

For each 3D ultrasound image, 3D SIFT-Rank features were extracted and matched as explained in the "Methods" section. Figure 4 shows a collection of 3D SIFT-Rank features extracted from iUS acquired before opening the dura membrane.

Figure 5 shows examples of feature correspondences between iUS images acquired before opening the dura membrane and prior to iMR (after partial resection).

Table 2 shows the number of 3D SIFT-Rank feature correspondences found in each pair of 3D ultrasound images. In this paper, pre-dura, post-dura, intraoperative and pre-iMR ultrasound images correspond to intraoperative ultrasound images acquired before opening the dura, after opening the dura, during tumor resection and prior to iMR, respectively.

Feature extraction and matching is a completely automated process that takes less than $30 \mathrm{~s}$ on average to extract and match features between two 3D ultrasound images using a 2.5 $\mathrm{GHz}$ Intel Core 2 processor. Virtually, all feature-based alignment running time is due to Gaussian convolution during feature extraction, and this represents a one-time preprocessing step that could be significantly reduced via GPU optimization. With features extracted, feature-based alignment requires less than $1 \mathrm{~s}$ using the same processor.

\section{Feature-based registration}

3D Slicer software was used for image visualization and registration [51]. Figure 6 shows the alignment between the pre-resection and post-resection (prior to intraoperative MR) ultrasound images before registration and after nonlinear correction. 


\section{Evaluation}

\section{Validation of SIFT-Rank correspondences via manual labeling}

SIFT-Rank features were presented in the pre-resection volume. Each expert was then asked to manually locate the correspondence for pre-resection feature in a second volume that was acquired at a different stage of the surgery. The discrepancy between manually and automatically located correspondences in the second volume can thus be used to quantify the accuracy of automatic correspondence, where a low discrepancy would indicate high agreement between automatic and manual correspondence. A user interface for the manual validation was built using 3D Slicer [51]. The 3D Euclidean distance between manual labels in the second volume was used to determine the inter-rater variability. The 3D Euclidean distance between the SIFT-Rank feature and the manual labels in the second volume was determined to know the discrepancy between SIFT-Rank features and manual labels.

\section{Registration validation using manual tags}

The ultrasound-ultrasound alignment was defined by selecting corresponding anatomical landmarks in the ultrasound images. Registration accuracy was measured as a function of the distance between these landmarks before and after registration [52]. We use mean target registration error (mTRE) metric, which shows the average distance between corresponding landmarks. Let $V_{1}$ and $V_{2}$ represent two ultrasound volumes and $x$ and $x^{\prime}$ represent corresponding landmarks in the $V_{1}$ and $V_{2}$ respectively, then mTRE can be calculated by Eq. (2).

$$
\text { mTRE }=\frac{1}{n} \sum_{i}^{n}\left\|T\left(x_{i}\right)-x^{\prime}{ }_{i}\right\|
$$

where $T$ is the deformation and $n$ is the number of landmarks.

\section{Qualitative registration evaluation by neurosurgeons}

The quality of the alignment of the pre- and post-resection ultrasound volumes was visually assessed by 2 physicians with 2 and 5 years of experience in medical imaging. Cross sections of the post-resection ultrasound volume were overlaid on (1) the original preresection ultrasound, (2) the pre-resection ultrasound after affine transform and (3) the preresection ultrasound after thin-plate spline. The experts accessed the registration accuracy at (1) anatomical landmarks such as the sulcal patterns, vessels, choroid plexus, falx and configuration of ventricles and (2) the tumor boundary to subjectively determine quality of registration with "bad" reflecting grossly visible misregistration, "good" reflecting minor visible misalignments, and "great" reflecting near undetectable misregistration.

\section{Other publicly available datasets}

Our registration framework was also validated using pre- and post-resection ultrasound images from the BITE [53] and RESECT [54] publicly available databases. Previous works have reported non-rigid registration results on intraoperative 3D ultrasound using BITE: 
RESOUND [23] presents a mTRE equal to $1.5(0.5-3.0) \mathrm{mm}$, and, more recent, NSR (nonrigid symmetric registration) [15] reported a mTRE equal to $1.5(0.4-3.1) \mathrm{mm}$.

\section{Results}

\section{Validation of SIFT-Rank correspondences via manual labeling}

A total of 1620 correspondences were manually validated by eleven experts, including medical imaging experts, radiologists and non-radiologist physicians. Figure 7 shows the manual validation of feature correspondences for pre- and post-resection ultrasound, where 71 feature correspondences were found. Given an automatically detected feature (circled in green), the experts were asked to locate the corresponding point in the second ultrasound volume (circled in orange).

For each feature correspondence, the 3D Euclidean distance was calculated between automatic and manually located features in the second volume. This distance measures the discrepancy between manually determined locations and automatically identified feature locations. This experiment was performed by 11 experts; each expert was randomly assigned a set of features to validate, and every feature correspondence was validated by at least 2 experts. The median, maximum and minimum distances were calculated on a per-feature basis, and the mean distance was calculated across all features. Figure 8 shows the mean feature-expert distance, where each column represents feature-expert distances for all feature correspondences between a specific pair of ultrasound volumes. The average target localization distance across all patients was equal to $2.20 \pm 0.43 \mathrm{~mm}$. The inter-rater variability was found to be equal to $1.89 \pm 0.37 \mathrm{~mm}$.

A second experiment was performed where we presented a set of experts with the automatic feature from the first volume and then both the automatic and manual matched features in the second volume. A total of 800 feature correspondences were used in this experiment. The experts were asked to choose which one was a better match. To avoid bias, the experts were blinded to which of the displayed correspondences were identified via SIFT-Rank and which were manually specified correspondences. We found that experts preferred the automatically detected features over the manually located features $88 \%$ of the time.

\section{Registration validation using manual tags}

For each patient, 10 unique landmarks were identified. Eligible landmarks include deep grooves and corners of sulci, convex points of gyri and vanishing points of sulci. Table 3 presents the number of landmarks and the mean, maximum and minimum initial 3D euclidean distance between landmark pairs. The mean, maximum and minimum distance after 3D SIFT-Rank-based affine and thin-plate spline are also presented.

The average initial mTRE for the 9 patients is $3.25 \mathrm{~mm}$. This value was reduced to $1.75 \mathrm{~mm}$ using SIFT-Rank-based affine and to $1.54 \mathrm{~mm}$ using SIFT-Rank-based thin-plate spline. To determine whether the mean distances were statistically different, a variance analysis (ANOVA) was applied. This yielded $F(1,32)=56.69, p<.001$, indicating that the differences were significant. There was also a statistically significant improvement between the initial displacements and after the SIFT-based thin-plate spline $(p<.001)$. 


\section{Qualitative registration evaluation by neurosurgeons}

For the 9 clinical cases, the neurosurgeons agree that the affine transform achieved acceptable registration in regions such as the falx and sulci (where deformation is expected to be small compared to the resection area) and thin-plate spline gave a good first approximation of the tumor boundary deformation. From a total of 20 pairs of ultrasound images, the alignment of 8 cases was classified as "good" and 12 cases as "great" after affine transform. After thin-plate spline, 4 cases were classified as "good" and 16 cases were classified as "great."

\section{Other publicly available datasets}

Our registration framework was also validated using pre- and post-resection ultrasound images from the BITE [53] and RESECT [54] publicly available databases. Tables 4 and 5 provide details on number of manually located anatomical landmarks of each dataset, and the mean, maximum and minimum initial 3D Euclidean distance between landmark pairs. The mean, maximum and minimum 3D Euclidean distance after SIFT-Rank-based affine and thin-plate spline are also presented. For each ultrasound image pair from BITE, 10 homologous landmarks were manually located. The number of 3D SIFT-Rank feature correspondences used to guide the registration per 3D ultrasound image pair is also presented in the second column of Table 4.

The average initial mTRE value over the 13 patients from BITE dataset is $3.59 \mathrm{~mm}$, which is reduced to $1.85 \mathrm{~mm}$ after SIFT-Rank-based affine and $1.52 \mathrm{~mm}$ after SIFT-Rank-based thinplate spline. To determine whether the mean distances were statistically different, a variance analysis (ANOVA) was applied. This yielded $F(1,24)=9.86, p<.001$, indicating a statistically significant improvement between the initial and the nonlinear transformation.

Our registration framework was also validated using pre-and post-resection ultrasound images from the RESECT [54] database.

The average initial mTRE value over the 17 patients from RESECT dataset was $3.54 \mathrm{~mm}$. This was reduced to $1.54 \mathrm{~mm}$ after SIFT-based affine and $1.49 \mathrm{~mm}$ after SIFT-based thinplate spline deformation. To determine whether the mean distances were statistically different, a variance analysis (ANOVA) was applied. This yielded $F(1,32)=20.42, p<.001$ indicating a statistically significant improvement between the initial and the nonlinear transformation.

\section{Conclusions}

We presented an efficient registration method for 3D iUS images achieved from a sparse set of automatically extracted feature correspondences. In this work, we focused mainly on intraoperative US registration in neurosurgery. The proposed methodology is particularly robust to missing or unrelated image structure and produces a set of feature correspondences that can be used to initialize more detailed and dense ultrasound registration. 3D SIFT-Rank features were shown to provide reasonable non-rigid registration of 3D iUS images while requiring low computational time for features extraction and matching. We found that our registration algorithm reduces the mTRE from an initial value of 3.3 to $1.5 \mathrm{~mm}(n=9)$. Our 
long-term goal is to use non-rigid registration to map pre-surgical image data (e.g., presurgical MR) to intra-operative image data (e.g., iUS or MR) to correct for brain tissue deformations that arise from brain shift and surgical interventions.

\section{Acknowledgments}

Funding This study is supported by the National Institute of Health Grants P41-EB015898-09, P41-EB015902 and R01-NS049251 and the Natural Sciences and Engineering Research Council of Canada, Discovery grant. The authors would like to acknowledge the financial support from the Portuguese Foundation for Science and Technology under the references PD/BD/105869/2014 and IDMEC/LAETA UID/EMS/50022/2013.

\section{References}

1. Bucholz RD, Smith KR, Laycock KA, McDurmont LL. Three-dimensional localization: from image-guided surgery to information-guided therapy. Methods. 2001; 25(2):186-200. [PubMed: 11812205]

2. Hill DG, Maurer CR, Maciunas RJ, Barwise JA, Fitzpatrick JM, Wang MY. Measurement of intraoperative brain surface deformation under a craniotomy. Neurosurgery. 1998; 43(3):514-528. [PubMed: 9733307]

3. Roberts D, Hartov A, Kennedy F, Miga M, Paulsen K. Intra-operative brain shift and deformation: a quantitative analysis of cortical displacement in 28 cases. Neurosurgery. 1998; 43:749-758. [PubMed: 9766300]

4. Letteboer MMJ, Willems PW, Viergever MA, Niessen WJ. Brain shift estimation in image-guided neurosurgery using 3-D ultrasound. IEEE Trans Biomed Eng. 2005; 52(2):268-276. [PubMed: 15709664]

5. Audette MA, Siddiqi K, Ferrie FP, Peters TM. An integrated range-sensing, segmentation and registration framework for the characterization of intra-surgical brain deformations in image-guided surgery. Comput Vis Image Underst. 2003; 89(2-3):226-251.

6. Marko NF, Weil RJ, Schroeder JL, Lang FF, Suki D, Sawaya RE. Extent of resection of glioblastoma revisited: personalized survival modeling facilitates more accurate survival prediction and supports a maximum-safe-resection approach to surgery. J Clin Oncol. 2014; 32(8):774. [PubMed: 24516010]

7. Coburger J, Merkel A, Scherer M, Schwartz F, Gessler F, Roder C, Jungk C. Low-grade glioma surgery in intraoperative magnetic resonance imaging: results of a multicenter retrospective assessment of the German Study Group for Intraoperative Magnetic Resonance Imaging. Neurosurgery. 2015; 78(6):775-786.

8. Brown TJ, Brennan MC, Li M, Church EW, Brandmeir NJ, Rakszawski KL, Glantz M. Association of the extent of resection with survival in glioblastoma: a systematic review and meta-analysis. JAMA Oncol. 2016; 2(11):1460-1469. [PubMed: 27310651]

9. Almeida JP, Chaichana KL, Rincon-Torroella J, Quinones-Hinojosa A. The value of extent of resection of glioblastomas: clinical evidence and current approach. Curr Neurol Neurosci Rep. 2015; 15(2):517. [PubMed: 25467408]

10. Hatiboglu MA, Weinberg JS, Suki D, Rao G, Prabhu SS, Shah K, Jackson E, Sawaya R. Impact of intra-operative high-field magnetic resonance imaging guidance on glioma surgery: a prospective volumetric study. Neurosurgery. 2009; 64:1073-1081. [PubMed: 19487886]

11. Claus EB, Horlacher A, Hsu L, Schwartz RB, Dello-Iacono D, Talos F, Jolesz FA, Black PM. Survival rates in patients with low-grade glioma after intra-operative magnetic resonance image guidance. Cancer. 2005; 103:1227-1233. [PubMed: 15690327]

12. Nabavi A, Black PM, Gering DT, Westin CF, Mehta V, Pergolizzi RS Jr, Ferrant M, Warfield SK, Hata N, Schwartz RB, Wells WM, Kikinis R, Jolesz F. Serial intra-operative magnetic resonance imaging of brain shift. Neurosurgery. 2001; 48:787-797. [PubMed: 11322439]

13. Kuhnt D, Bauer MH, Nimsky C. Brain shift compensation and neurosurgical image fusion using intraoperative MRI: current status and future challenges. Crit Rev Biomed Eng. 2012; 40:175-185. [PubMed: 22694198] 
14. Tirakotai D, Miller S, Heinze L, Benes L, Bertalanffy H, Sure U. A novel platform for imageguided ultrasound. Neuro-surgery. 2006; 58:710-718.

15. Zhou H, Rivaz H. Registration of pre-and postresection ultrasound volumes with noncorresponding regions in neurosurgery. IEEE J Biomed Health Inform. 2016; 20(5):1240-1249. [PubMed: 27101626]

16. Rivaz H, Collins DL. Near real-time robust non-rigid registration of volumetric ultrasound images for neurosurgery. Ultrasound Med Biol. 2015; 41(2):574-587. [PubMed: 25542482]

17. Mercier L, Araujo D, Haegelen C, Del Maestro RF, Petrecca K, Collins DL. Registering pre- and post-resection 3-dimensional ultrasound for improved visualization of residual brain tumor. Ultrasound Med Biol. 2013; 39(1):16-29. [PubMed: 23200177]

18. Gerard IJ, Kersten-Oertel M, Petrecca K, Sirhan D, Hall JA, Collins DL. Brain shift in neuronavigation of brain tumors: a review. Med Image Anal. 2017; 35:403-420. [PubMed: 27585837]

19. Pheiffer TS, Thompson RC, Rucker DC, Simpson AL, Miga MI. Model-based correction of tissue compression for tracked ultrasound in soft tissue image-guided surgery. Ultrasound Med Biol. 2014; 40(4):788-803. [PubMed: 24412172]

20. Morin F, Chabanas M, Courtecuisse H, Payan Y. Biomechanics of living organs. Academic Press; Cambridge: 2017. Biomechanical modeling of brain soft tissues for medical applications; 127 146.

21. Morin F, Courtecuisse H, Reinertsen I, Lann FL, Palombi O, Payan Y, Chabanas M. Brain-shift compensation using intraoperative ultrasound and constraint-based biomechanical simulation. Med Image Anal. 2017; 40:133-153. [PubMed: 28651099]

22. Luo M, Frisken SF, Weis JA, Clements LW, Unadkat P, Thompson RC, Golby AJ, Miga MI. Validation of model-based brain shift correction in neurosurgery via intraoperative magnetic resonance imaging: preliminary results. Proceedings of SPIE 10135, medical imaging: imageguided procedures, robotic interventions, and modeling; 2017.

23. Rivaz H, Collins DL. Deformable registration of preoperative MR, pre-resection ultrasound, and post-resection ultrasound images of neurosurgery. Int J Comput Assist Radiol Surg. 2015; 10(7): 1017-1028. [PubMed: 25373447]

24. Blumenthal T, Hartov A, Lunn K, Kennedy FE, Roberts DW, Paulsen KD. Quantifying brain shift during neurosurgery using spatially tracked ultrasound. Proceedings of SPIE 5744, medical imaging: visualization, image-guided procedures, and display; 2005.

25. Mercier L, Fonov V, Haegelen C, Del Maestro RF, Petrecca K, Collins DL. Comparing two approaches to rigid registration of three-dimensional ultrasound and magnetic resonance images for neurosurgery. Int J Comput Assist Radiol Surg. 2012; 7(1):125-136. [PubMed: 21633799]

26. Poon T, Rohling R. Three-dimensional extended field-of-view ultrasound. Ultrasound Med Biol. 2006; 32:357-369. [PubMed: 16530094]

27. Schers J, Troccaz J, Daanen V, Fouard C, Plaskos C, Kilian P. 3D/4D ultrasound registration of bone. Proceedings of the IEEE ultrasonics symposium; 2007. 2519-2522.

28. Comaniciu D, Meer P. Mean shift: a robust approach toward feature space analysis. IEEE TPAMI. 2002; 24(5):603-619.

29. Grimson WEL, Lozano-Perez T. Localizing overlapping parts by searching the interpretive tree. IEEE TPAMI. 1987; 9(4):469-482.

30. Beis JS, Lowe DG. Shape indexing using approximate nearest-neighbour search in highdimensional spaces. CVPR. 1997:1000-1006.

31. Biederman I. Recognition-by-components: a theory of human image understanding. Psychol Rev. 1987; 2(94):115-147.

32. Pratikakis I. Robust multiscale deformable registration of 3D ultrasound images. Int J Image Graph. 2003; 3:547-565.

33. Cen F, Jiang Y, Zhang Z, Tsui HT, Lau TK, Xie H. Robust registration of 3D-ultrasound images based on gabor filter and mean-shift. Method. 2004:304-316.

34. Schneider RJ, Perrin DP, Vasilyev NV, Marx GR, Pedro J, Howe RD. Real-time image-based rigid registration of three-dimensional ultrasound. Med Image Anal. 2012; 16:402-414. [PubMed: 22154960] 
35. Toews M, Wells WM III. Efficient and robust model-to-image registration using 3D scale-invariant features. Med Image Anal. 2013; 17(3):271-282. [PubMed: 23265799]

36. Ni D, Qu Y, Yang X, Chui YP, Wong TT, Ho SS, Heng PA. Volumetric ultrasound panorama based on 3D SIFT. International conference on medical image computing and computer-assisted intervention; Berlin: Springer; 2008. 52-60.

37. Bersvendsen J, Toews M, Danudibroto A, Wells WM, Urheim S, Estépar RSJ, Samset E. Medical imaging 2016: ultrasonic imaging and tomography. Vol. 9790. International society for optics and photonics; 2016. Robust spatio-temporal registration of 4D cardiac ultrasound sequences; 97900F

38. Kadir T, Brady M. Saliency, scale and image description. Int J Comput Vis. 2001; 45(2):83-105.

39. Mikolajczyk K, Schmid C. A performance evaluation of local descriptors. IEEE Trans Pattern Anal Mach Intell. 2005; 27(10):1615-1630. [PubMed: 16237996]

40. Toews M, Wells WM. Sift-rank: ordinal description for invariant feature correspondence. IEEE conference on computer vision and pattern recognition, 2009. CVPR 2009; 2009. 172-177.

41. Jenkinson M, Smith S. A global optimisation method for robust affine registration of brain images. Med Image Anal. 2001; 5(2):143-156. [PubMed: 11516708]

42. Bookstein FL. Principal warps: thin-plate splines and the decomposition of deformations. IEEE Trans Pattern Anal Mach Intell. 1989; 11(6):567-585.

43. Rohr K, Stiehl HS, Sprengel R, Beil W, Buzug TM, Weese J, Kuhn MH. Visualization in biomedical computing. Springer; Berlin: 1996. Point-based elastic registration of medical image data using approximating thin-plate splines; 297-306.

44. Tempany C, Jayender J, Kapur T, Bueno R, Golby A, Agar N, Jolesz FA. Multimodal imaging for improved diagnosis and treatment of cancers. Cancer. 2015; 121(6):817-827. [PubMed: 25204551]

45. Strong EB, Rafii A, Holhweg-Majert B, Fuller SC, Metzger MC. Comparison of 3 optical navigation systems for computer-aided maxillofacial surgery. Arch Otolaryngol Head Neck Surg. 2008; 134(10):1080-1084. [PubMed: 18936355]

46. Tokuda J, Fischer GS, Papademetris X, Yaniv Z, Ibanez L, Cheng P, Liu H, Blevins J, Arata J, Golby AJ, Kapur T, Pieper S, Burdette EC, Fichtinger G, Tempany CM, Hata N. OpenIGTLink: an open network protocol for image-guided therapy environment. Int J Med Robot. 2009; 5(4):42334. [PubMed: 19621334]

47. Lasso A, Heffter T, Rankin A, Pinter C, Ungi T, Fichtinger G. PLUS: open-source toolkit for ultrasound-guided intervention systems. IEEE Trans Biomed Eng. 2014; 61(10):2527-2537. [PubMed: 24833412]

48. Gobbi DG, Peters TM. Interactive intra-operative 3D ultrasound reconstruction and visualization. International conference on medical image computing and computer-assisted intervention; Berlin: Springer; 2002. 156-163.

49. Boisvert J, Gobbi D, Vikal S, Rohling R, Fichtinger G, Abolmaesumi P. An open-source solution for interactive acquisition, processing and transfer of interventional ultrasound images. The MIDAS journal-systems and architectures for computer assisted interventions. 2008:70.

50. Selbekk T, Jakola AS, Solheim O, Johansen TF, Lindseth F, Reinertsen I, Unsgård G. Ultrasound imaging in neurosurgery: approaches to minimize surgically induced image artefacts for improved resection control. Acta Neurochir. 2013; 155(6):973-980. DOI: 10.1007/s00701-013-1647-7 [PubMed: 23459867]

51. Kikinis R, Pieper SD, Vosburgh K. 3D Slicer: a platform for subject-specific image analysis, visualization, and clinical support. Intraoperative Imaging Image Guided Ther. 2014; 3(19):277_ 289.

52. Jannin P, Fitzpatrick JM, Hawkes D, Pennec X, Shahidi R, Vannier M. Validation of medical image processing in image-guided therapy. IEEE Trans Med Imaging. 2002; 21(12):1445-9. [PubMed: 12588028]

53. Mercier L, Del Maestro RF, Petrecca K, Araujo D, Haegelen C, Collins DL. Online database of clinical MR and ultrasound images of brain tumors. Med Phys. 2012; 39(6Part1):3253-3261. [PubMed: 22755708] 
54. Xiao Y, Fortin M, Unsgård G, Rivaz H, Reinertsen I. REtroSpective Evaluation of Cerebral Tumors (RESECT): a clinical database of pre-operative MRI and intra-operative ultrasound in low-grade glioma surgeries. Med Phys. 2017; 44(7):3875-3882. [PubMed: 28391601] 


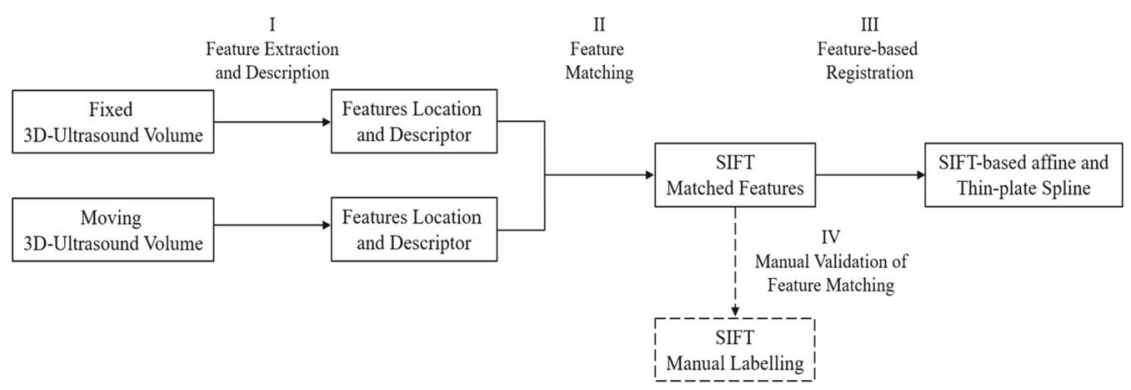

Fig. 1.

Summary of feature-based registration for intraoperative 3D ultrasound images 


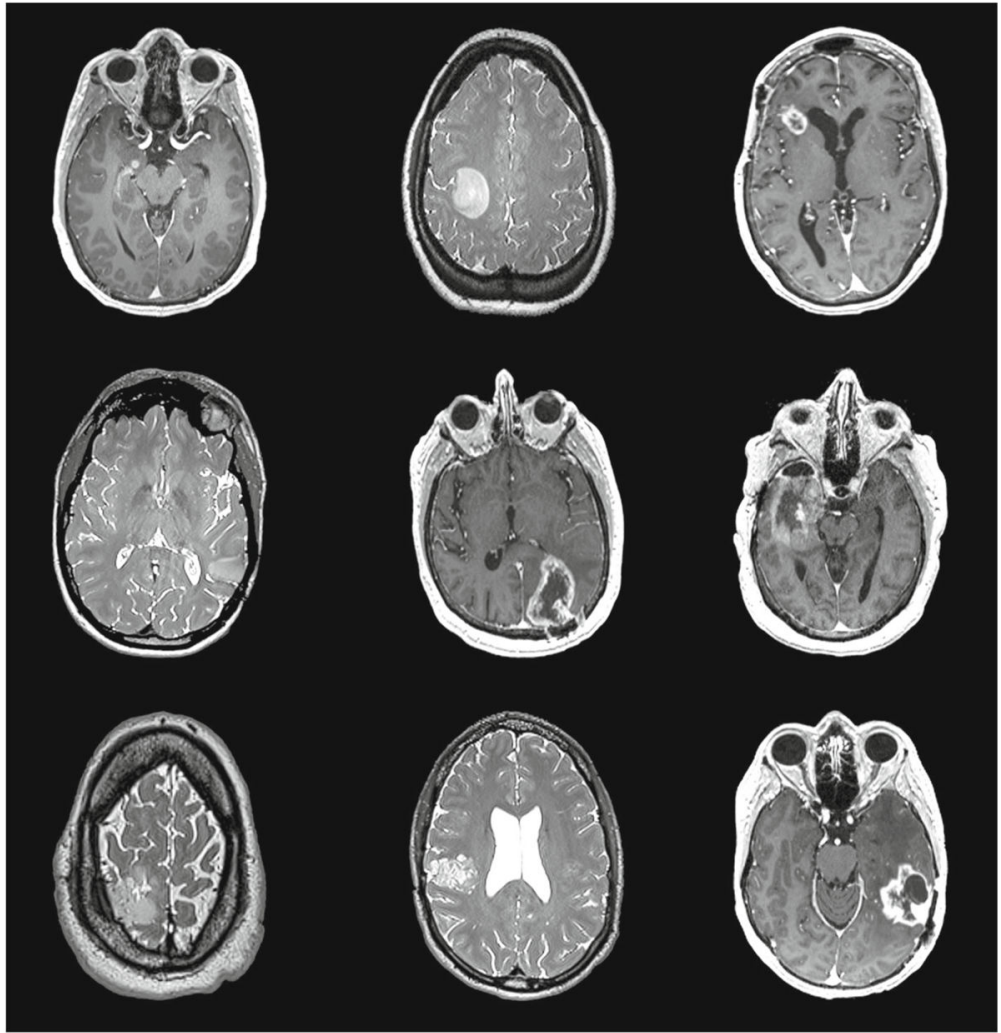

Fig. 2.

Axial slices of 9 preoperative MR subject images showing tumor locations

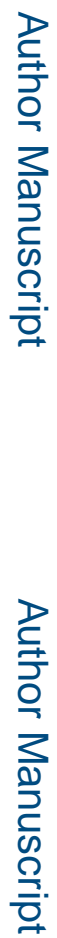




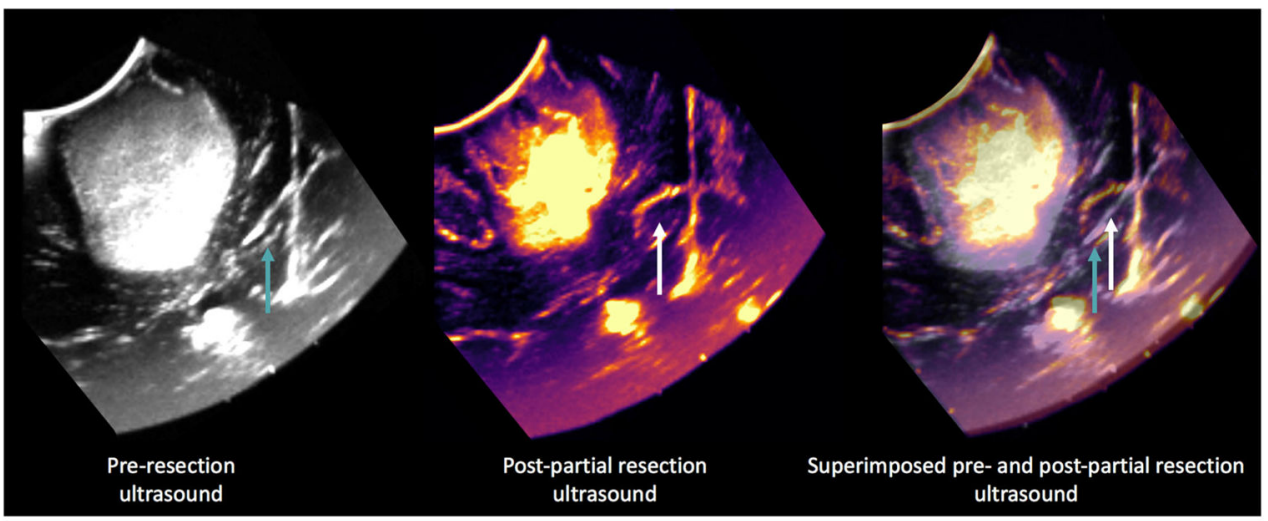

Fig. 3.

Pre-resection (gray shades) and post-partial resection (warm shades) ultrasound images. Arrows indicate the falx and show its misalignment in images taken at different time points (far right) 

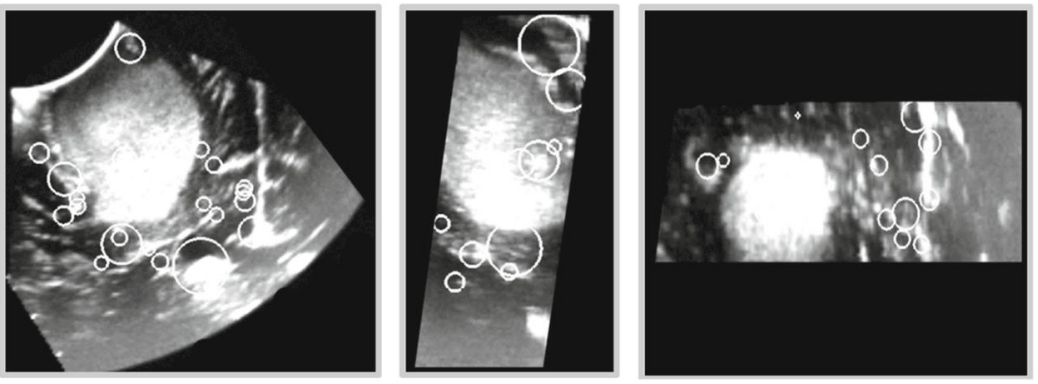

Fig. 4.

3D SIFT-Rank features of a 3D ultrasound image representing a right-frontal anaplastic astrocytoma. Axial, sagittal and coronal views are presented from the left to the right. Each white circle overlaying the image represents the location $\bar{x}$ and scale $\sigma$ of an automatically detected local feature 

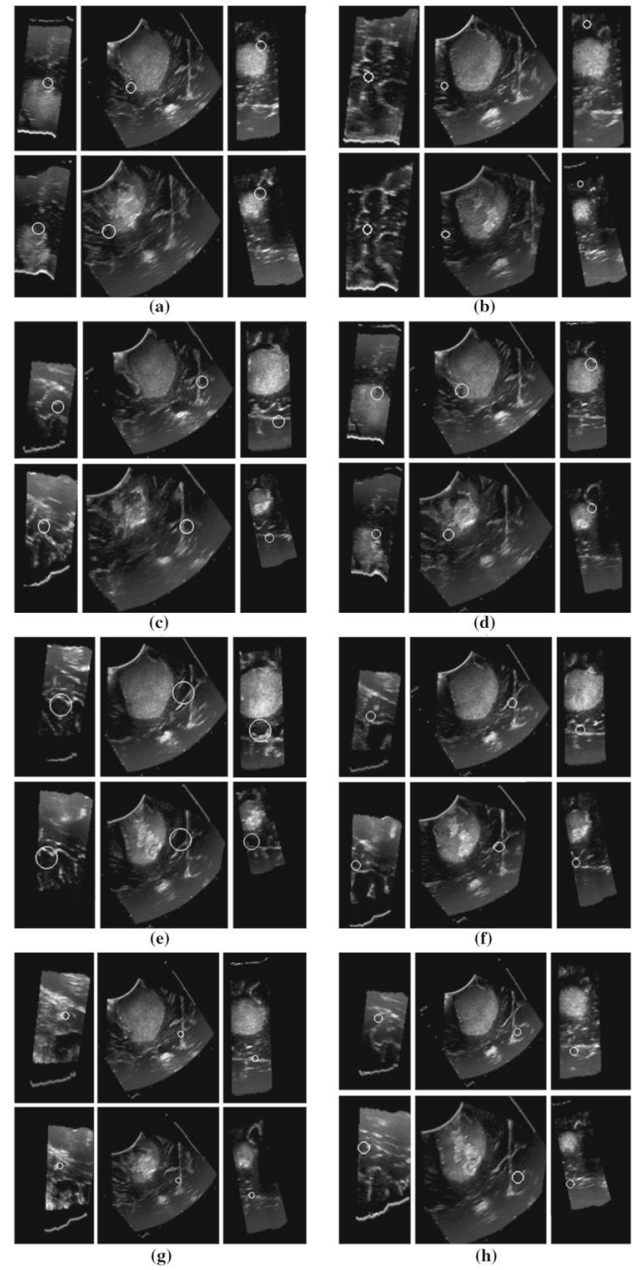

Fig. 5.

3D SIFT-Rank feature correspondences in a right-frontal anaplastic astrocytoma between 3D ultrasound images. In each of $\mathbf{a}-\mathbf{h}$ white circles represent the local and scale of a single scale-invariant feature in three orthogonal image slices, automatically identified in two different ultrasound volumes (upper and lower triplets). Note the high degree of visual similarity in the upper and lower images, surrounding the feature of interest 


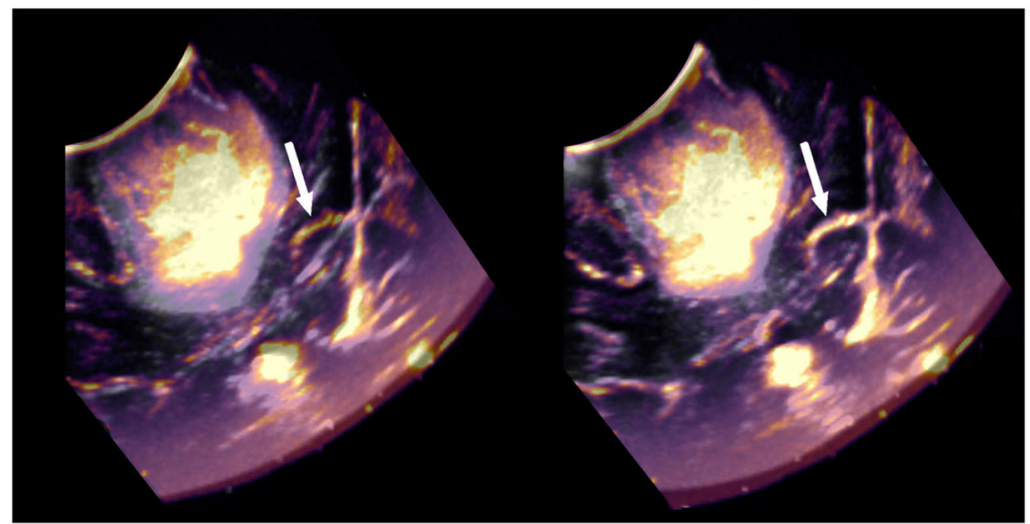

Fig. 6.

Pre-resection (gray shades) and post-resection (warm shades) ultrasound images. A typical example of pre- and post-resection ultrasound images and their initial misalignments is shown on the left. Alignment after SIFT-Rank-based thin-plate spline (on the right) between two iUS images 

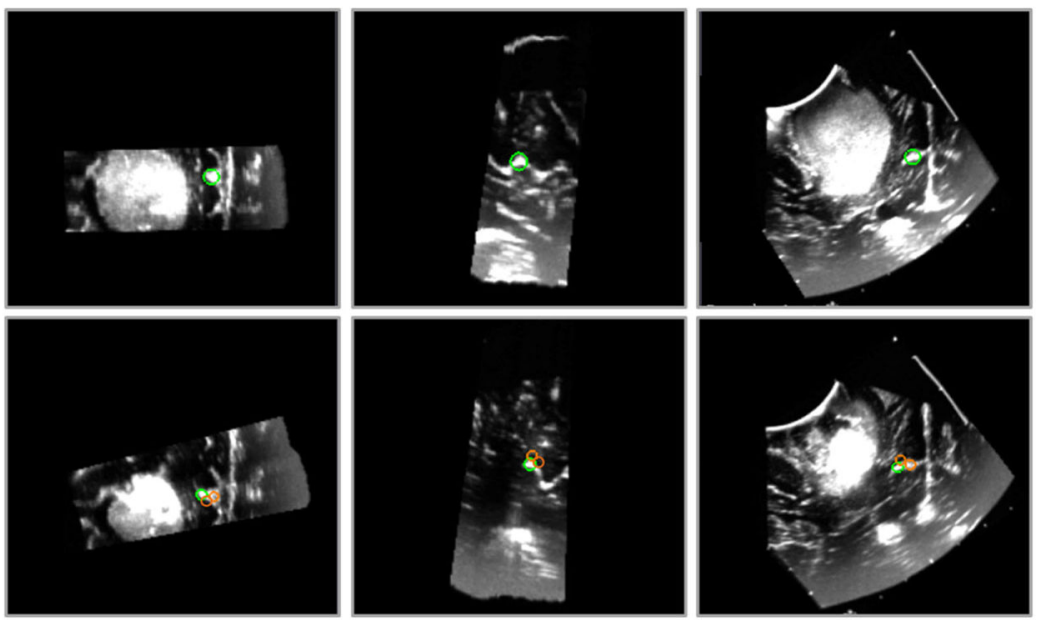

Fig. 7.

3D SIFT-Rank features manual validation. The orange circles represent the locations of a feature as estimated manually by different human experts, and the green circles represent the location identified via automatic SIFT-Rank features matching. The upper row corresponds to three different views of the pre-dura volume, and the lower row corresponds to an ultrasound volume acquired prior to iMR 


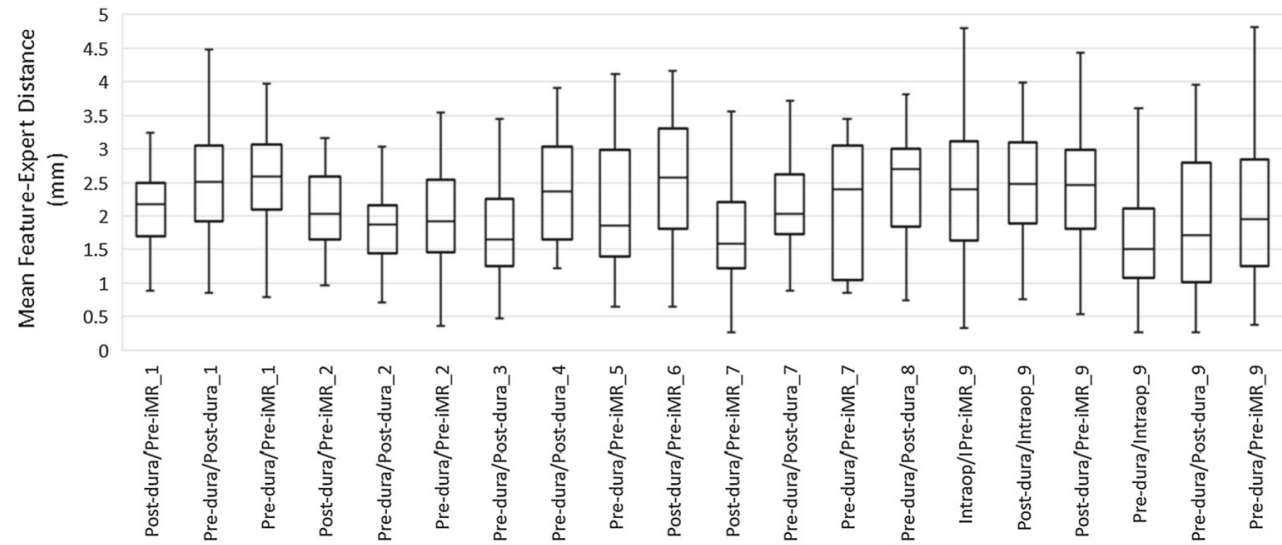

Fig. 8.

Feature-to-expert distances $(\mathrm{mm})$ between each pair of ultrasound volumes and across different patients 


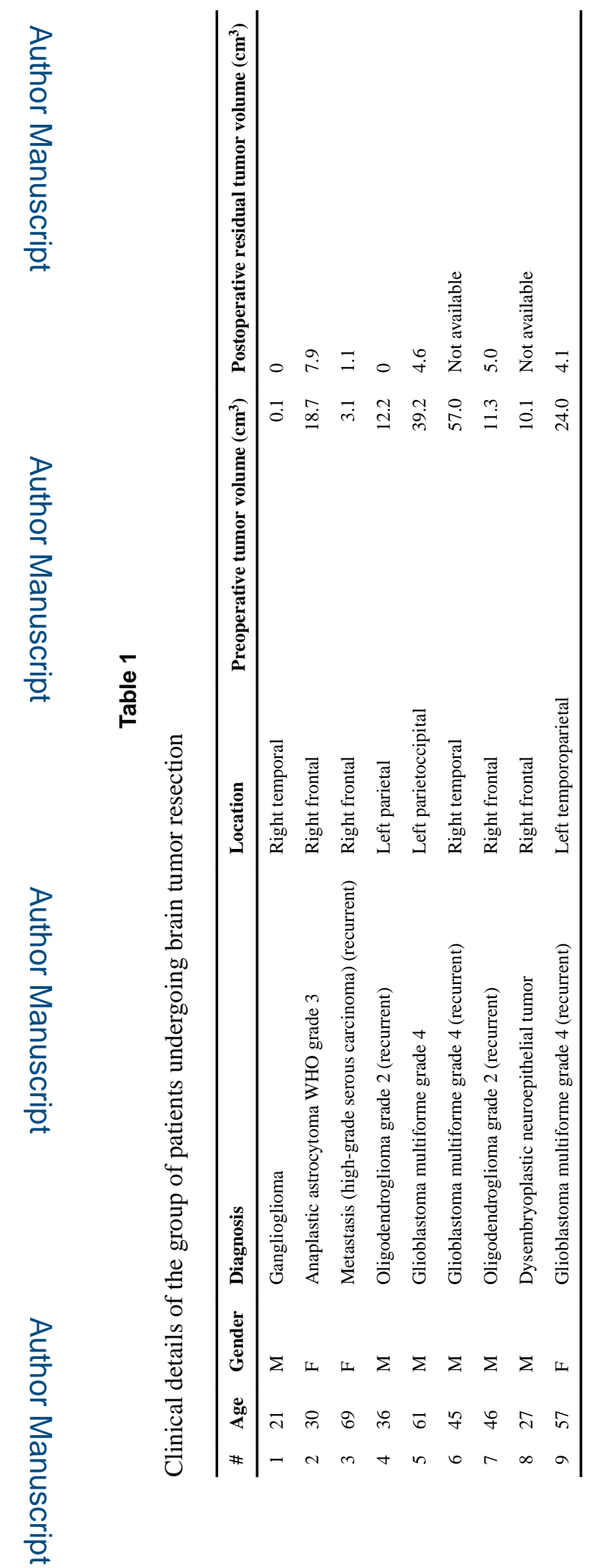

Int J Comput Assist Radiol Surg. Author manuscript; available in PMC 2019 October 01. 
Table 2

Number of 3D SIFT-Rank feature correspondences on pairs of ultrasound volumes acquired at different time points during surgery

\begin{tabular}{llll}
\hline US data & Patient 1 & Patient 2 & Patient 7 \\
\hline Post-dura US/pre- iMR US & 81 & 22 & 46 \\
Pre-dura US/pre-iMR US & 123 & 71 & 12 \\
Pre-dura US/post-dura US & 124 & 57 & 30 \\
\hline US Data & Patient 3 & Patient 4 & Patient 8 \\
\hline Pre-dura US/post-dura US & \multirow{2}{*}{84} & 14 & 37 \\
\hline US Data & & \multirow{2}{*}{ Patient 5 } & Patient 6 \\
\hline Pre-dura US/pre-iMR US & & 49 & N/A \\
Post-dura US/pre-iMR US & & N/A & 98 \\
\hline US Data & & & Patient 9 \\
\hline Intraop US/pre-iMR US & & & 250 \\
Post-dura US/pre-iMR US & & & 123 \\
Post-dura US/intraop US & & & 133 \\
Pre-dura US/pre-iMR US & & & 50 \\
Pre-dura US/intraop US & & & 151 \\
Pre-dura US/post-dura US & & & \\
\hline
\end{tabular}




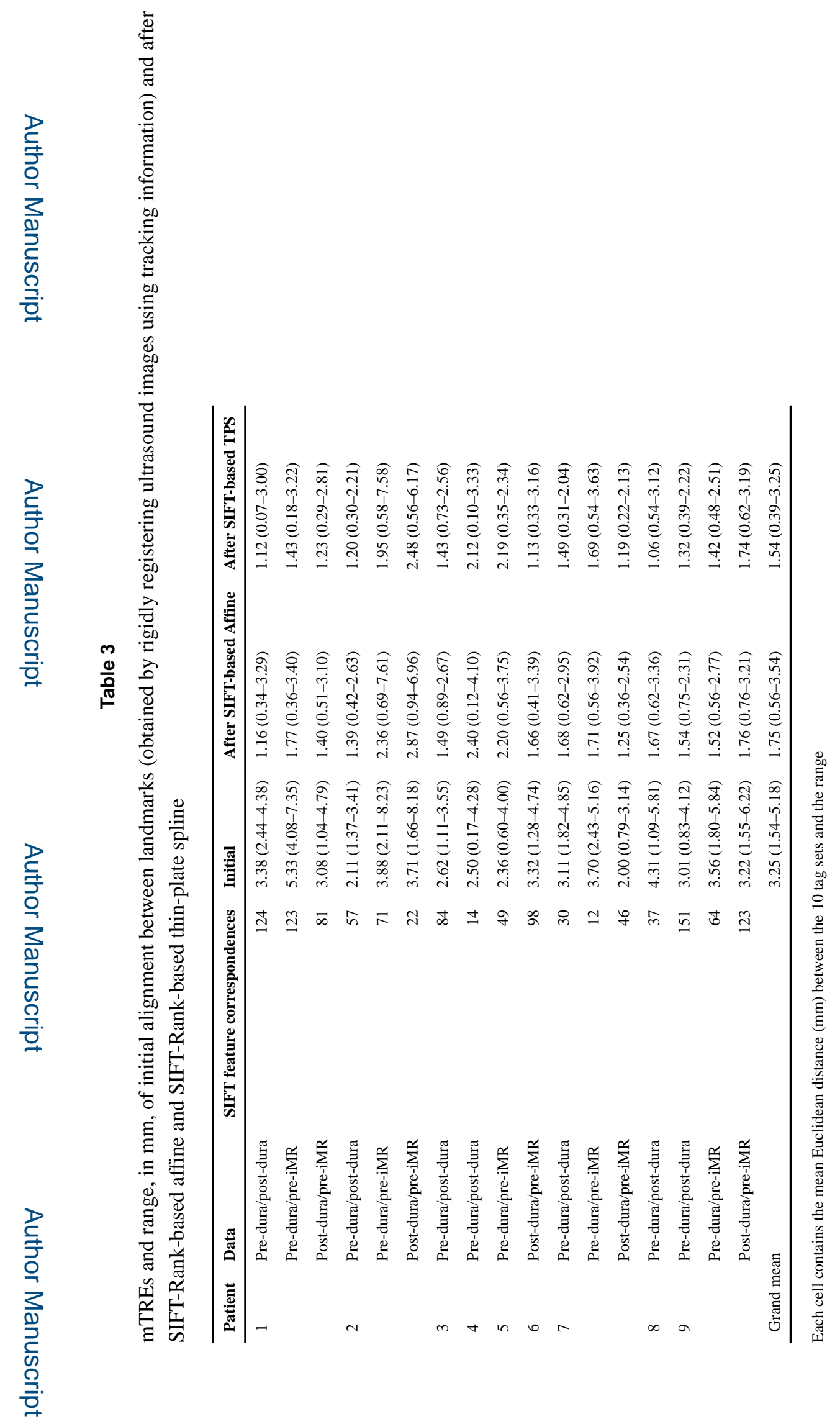

Int J Comput Assist Radiol Surg. Author manuscript; available in PMC 2019 October 01. 


\section{Table 4}

BITE database: mTREs and range, in mm, of initial alignment between landmarks and after SIFT-Rank-based affine and SIFT-Rank-based thin-plate spline

\begin{tabular}{lclll}
\hline Patient & SIFT feature correspondences & Initial & After SIFT-based affine & After SIFT-based TPS \\
\hline 1 & No post-resection US & & & \\
2 & 54 & $2.30(0.57-5.42)$ & $1.80(0.39-5.16)$ & $1.66(0.38-4.07)$ \\
3 & 55 & $3.78(2.80-5.09)$ & $1.31(0.63-2.33)$ & $1.30(0.38-2.15)$ \\
4 & 26 & $4.60(2.96-5.88)$ & $1.26(0.53-3.00)$ & $1.13(0.42-2.74)$ \\
5 & 27 & $4.11(2.58-5.26)$ & $1.24(0.59-1.77)$ & $1.24(0.50-1.70)$ \\
6 & 183 & $2.26(1.36-3.10)$ & $1.33(0.63-2.34)$ & $1.18(0.59-2.11)$ \\
7 & 58 & $3.87(2.60-5.07)$ & $1.39(0.56-2.31)$ & $0.86(0.39-1.38)$ \\
8 & 29 & $2.51(0.67-3.93)$ & $2.15(0.60-3.83)$ & $1.12(0.49-3.08)$ \\
9 & 19 & $2.21(1.00-4.59)$ & $2.03(0.70-3.95)$ & $1.38(0.44-3.71)$ \\
10 & 45 & $3.86(0.98-6.68)$ & $2.68(0.78-5.65)$ & $2.59(0.72-3.51)$ \\
11 & 82 & $2.88(0.76-8.95)$ & $2.87(0.74-7.95)$ & $2.52(0.48-3.23)$ \\
12 & 67 & $10.53(7.85-13.06)$ & $3.68(0.67-8.06)$ & $2.37(0.14-3.74)$ \\
13 & 93 & $1.62(1.33-2.21)$ & $1.04(0.53-2.76)$ & $1.01(0.29-2.03)$ \\
14 & 188 & $2.19(0.59-3.99)$ & $1.32(0.27-2.66)$ & $1.04(0.25-2.40)$ \\
Grand means & & $3.59(2.00-5.63)$ & $1.85(0.59-3.98)$ & $1.52(0.42-2.76)$ \\
\hline
\end{tabular}




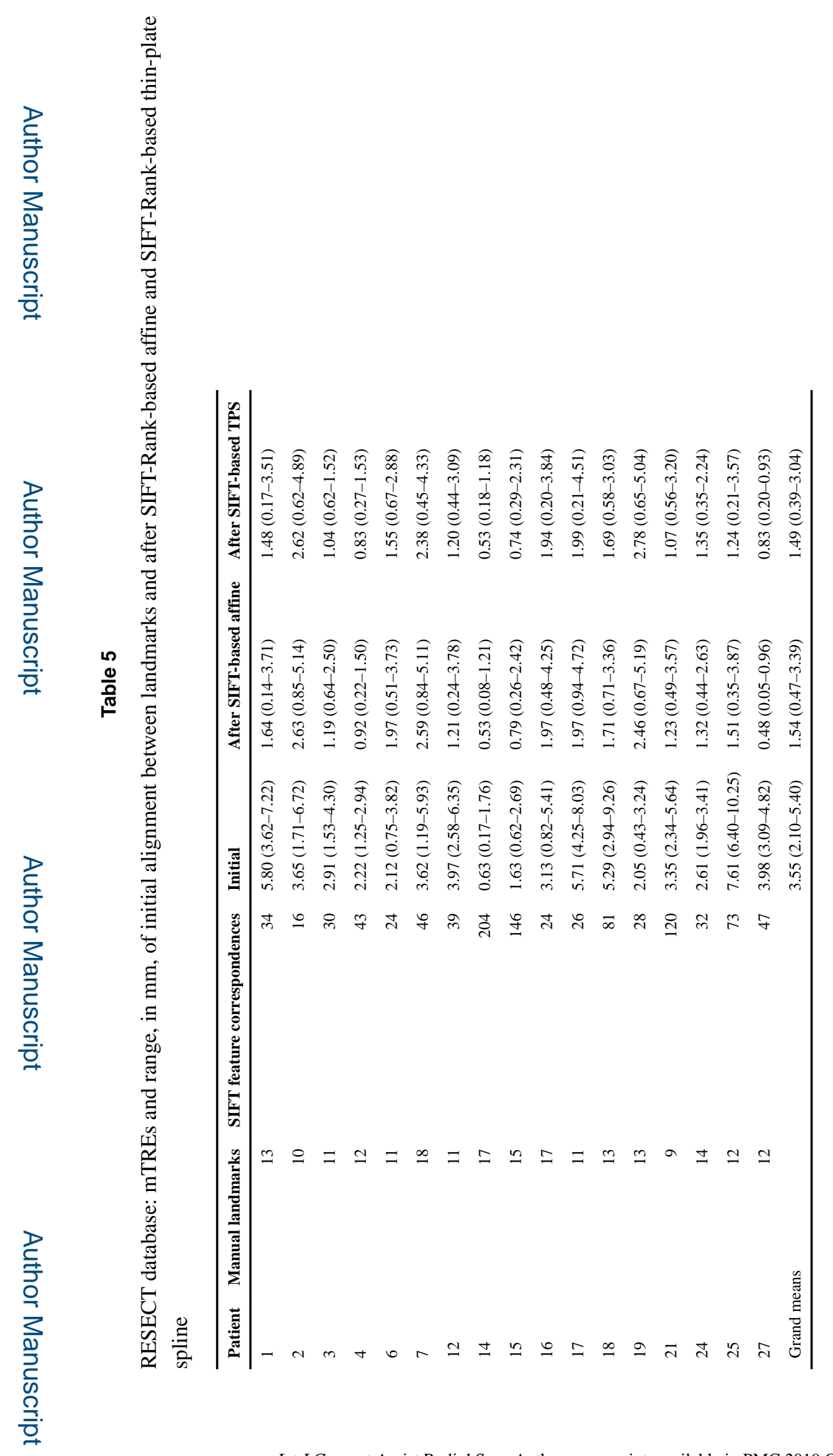

Int J Comput Assist Radiol Surg. Author manuscript; available in PMC 2019 October 01. 